\title{
Participation End Date
}

National Cancer Institute

\section{Source}

National Cancer Institute. Participation End Date. NCI Thesaurus. Code C162172.

The date of the end of a subject's participation in the study. 